\title{
Aplicação da escala de coma de Glasgow: uma analise bibliométrica acerca das publicações no âmbito da Enfermagem
}

\author{
Application of the Glasgow coma scale: a bibliometric analysis of publications in the field of \\ Nursing \\ Aplicación de la escala de coma de Glasgow: análisis bibliométrico de publicaciones en el campo de \\ la Enfermería
}

Recebido: 11/10/2021 | Revisado: 16/10/2021 | Aceito: 22/10/2021 | Publicado: 24/10/2021

\author{
Luana Miranda de Sousa \\ ORCID: https://orcid.org/0000-0002-6283-6263 \\ Faculdade Integrada Carajás, Brasil \\ E-mail: luanamiranda2017@gmail.com \\ Marcos Vinícius Ferreira dos Santos \\ ORCID: https://orcid.org/0000-0003-1335-1021 \\ Faculdade Integrada Carajás, Brasil \\ E-mail: viniciosferreirasantos@hotmail.com
}

\begin{abstract}
Resumo
O artigo objetiva realizar uma analise bibliométrica de diferentes estudos acerca da Aplicação da Escala de Coma de Glasgow, a fim de levantar a melhor compreensão acerca da temática desse estudo. Para isso, foi realizado um estudo bibliométrico com base em pesquisas bibliográficas acerca da temática escala de Coma de Glasgow e aplicação da escala de Coma de Glasgow. Foram selecionados nove artigos, dos dezessete encontrados, com base na analise destes artigos foi possível identificar e alcançar o seu objetivo principal, que era realizar uma analise bibliométrica de diferentes estudos acerca da Aplicação da Escala de Coma de Glasgow, onde se pode identificar que esta é muito empregada hoje para identificar disfunções neurológicas, acompanhar a evolução do nível de consciência, predizer prognósticos e padronizar a linguagem entre os profissionais de saúde. Além do mais, a partir da seleção de 9 artigos verificou-se que $78 \%$ dos estudos pontuados alcançaram objetivos positivos demonstrado o conhecimento dos profissionais e as vantagens de aplicação da ECG. Já 11,11\% dos resultados foram insatisfatórios e/ou intermediários, ou seja, um somatório de $22,22 \%$ dos artigos pontuados obtiveram resultados negativos, o que leva ao entendimento de que em alguma aplicação o conhecimento da ECG se faz insatisfatório.
\end{abstract}

Palavras-chave: Escala de Glasgow; Enfermagem; Saúde; Paciente; Traumatismo cranioencefálico.

\begin{abstract}
The article aims to carry out a bibliometric analysis of different studies on the Application of the Glasgow Coma Scale, in order to raise a better understanding of the theme of this study. For this, a bibliometric study was carried out based on bibliographic research on the thematic Glasgow Coma Scale and application of the Glasgow Coma Scale. Nine articles were selected, from the seventeen found, based on the analysis of these articles it was possible to identify and achieve its main objective, which was to carry out a bibliometric analysis of different studies on the Application of the Glasgow Coma Scale, where it can be identified that this it is widely used today to identify neurological disorders, monitor the evolution of the level of consciousness, predict prognoses and standardize language among health professionals. Furthermore, from the selection of 9 articles, it was found that $78 \%$ of the scored studies achieved positive objectives, demonstrating the knowledge of professionals and the advantages of applying ECG. On the other hand, $11.11 \%$ of the results were unsatisfactory and/or intermediate, that is, a sum of 22, $22 \%$ of the scored articles had negative results, which leads to the understanding that, in some application, knowledge of ECG is unsatisfactory.
\end{abstract}

Keywords: Glasgow scale; Nursing; Health; Patient; Cranioencephalic trauma.

\section{Resumen}

El artículo tiene como objetivo realizar un análisis bibliométrico de diferentes estudios sobre la Aplicación de la Escala de Coma de Glasgow, con el fin de mejorar la comprensión de la temática de este estudio. Para ello se realizó un estudio bibliométrico basado en la investigación bibliográfica sobre la temática Escala de coma de Glasgow y aplicación de la Escala de coma de Glasgow. Se seleccionaron nueve artículos, de los diecisiete encontrados, a partir del análisis de estos artículos se pudo identificar y lograr su principal objetivo, que fue realizar un análisis bibliométrico de diferentes estudios sobre la Aplicación de la Escala de Coma de Glasgow, donde Se puede identificar 
que este es muy utilizado en la actualidad para identificar trastornos neurológicos, monitorear la evolución del nivel de conciencia, predecir pronósticos y estandarizar el lenguaje entre los profesionales de la salud. Además, de la selección de 9 artículos, se encontró que el $78 \%$ de los estudios puntuados lograron objetivos positivos, demostrando el conocimiento de los profesionales y las ventajas de aplicar el ECG. Por otro lado, el 11,11\% de los resultados fueron insatisfactorios y / o intermedios, es decir, una suma del 22,22\% de los artículos puntuados tuvieron resultados negativos, lo que lleva a entender que, en alguna aplicación, el conocimiento del ECG es insatisfactorio.

Palabras clave: Escala de Glasgow; Enfermería; Salud; Paciente; Traumatismo craneoencefálico.

\section{Introdução}

A escala de Glasgow foi desenvolvida e publicada na década de 1970, na revista Lancet, por dois neurologistas, Graham Teasdale e Bryan Jennett. Hoje se configura como a principal referencia para analise de nível de consciência em pacientes traumatizados, baseando-se na quantificação da resposta ocular, verbal e motora (Silva \& Cunha, 2020).

Esse método tem grande aplicabilidade visto que objetiva fornecer uma metodologia de atendimento que aponte a profundidade do dano neurológico quanto a duração clinica de inconsciência e coma, auxiliando dessa forma, no prognostico da vitima e na prevenção de possíveis seqüelas (Melo, et al, 2019).

Levando em consideração o quantitativo de traumas cranioencefálicos (TCE) no Brasil, e o seu índice de mortalidade, surge a necessidade de entender e investigar a gravidade desse tema. Tomando como base que a variabilidade da condição clínica das vítimas de TCE, nas primeiras horas pós-trauma, tem relação com as alterações fisiológicas que ocorrem em consequência da lesão anatômica, e os escores da Escala de Coma de Glasgow (ECGl) sumarizam as manifestações clínicas da evolução da lesão, torna-se importante analisar os estudos acerca dessa temática (Settervall; Sousa; Silva, 2011).

A partir dessas informações é necessário analisar se esta Escala vendo sendo empregada para identificar disfunções neurológicas, acompanhar a evolução do nível de consciência, predizer prognósticos e padronizar a linguagem entre os profissionais de saúde.

Dessa forma, o objetivo deste estudo é realizar uma analise bibliométrica de diferentes estudos acerca da Aplicação da Escala de Coma de Glasgow. Para atingir tal objetivo será apresentado as principais aplicações da Escala de Coma de Glasgow, apresentar a escala de coma de Glasgow e suas mudanças e fundamentar uma discussão com base na metanálise de dados, apresentando estes dados acerca de estudos de aplicação da Escala de Coma de Glasgow.

Objetivando a melhor compreensão acerca da temática esse estudo se organiza da seguinte forma: o referencial teórico aborda conceitos importantes para a compreensão geral da temática, tais quais a conceituação da Escala de Coma de Glasgow, os elementos da escala, seguidos da abordagem do traumatismo cranioencefálico e os cuidados, tratamos também sobre as mudanças ocorridas na escala de coma de Glasgow e suas formulas e pontuações.

Posteriormente são apresentados o enquadramento metodológico dessa pesquisa, em consonância com as etapas e procedimentos técnicos utilizados para o seu desenvolvimento. Em seguida apresentam-se os resultados e discussões acerca do estudo levantado, com base na Analise bibliométrica realizada e demonstrada na Analise dos dados.

Por fim, esse estudo traz as considerações finais da autora acerca da temática, pontuando a compreensão do que foi desenvolvido e deixando observações de oportunidades de futuras pesquisas.

\section{Referencial Teórico}

\subsection{Escala de coma de Glasgow}

A escala de Coma de Glasgow foi desenvolvida em meados de 1970, por dois neurologistas, Graham Teasdale e Bryan Jennett, utilizada para avaliações neurológicas a fim de determinar o nível de consciência e repostas motoras através do escore de sua escala. Silva e Cunha (2020) pontuam que a escala é empregada hoje para identificar disfunções neurológicas, 
acompanhar a evolução do nível de consciência, predizer prognósticos e padronizar a linguagem entre os profissionais de saúde.

O grau de consciência que uma pessoa apresenta torna-se um fator importante, quando está sendo avaliado o neurológico, pois através deste alerta comportamental é verificado qual estado à pessoa se encontra (Feijó, 2015). As possíveis variâncias em pacientes neurológicos deverão ser acompanhadas e mensuradas durante o tratamento.

Devem ser levados em consideração alguns pontos, como fatores eventuais, fatores de risco como a pontuação da ECG, avaliar também a incidência da necessidade de intubação orotraqueal, esses fatores são relevantes para que seja definida cada emergência e dados dentro de um hospital (Morgado, 2011).

A escala é uma prioridade do enfermeiro sendo avaliada a consciência através da tabela com as respostas transmitidas pelo paciente, o acompanhamento do pessoal da enfermagem, enquanto o paciente estiver alocado no hospital é essencial para que estejam muito cientes sobre o estado que o paciente realmente se encontra.

Os cuidados ocorridos nas primeiras 96 horas, ou seja, nos primeiros quatro dias de internação, são primordiais para notar a evolução do paciente com uma recuperação maior ou menor, das vitimas de AVC ou trauma cranioencefálico, se caso, nesse período constatado como crítico não for possível atuar, provavelmente será um paciente com graves sequelas ou poderá evoluir para o óbito (COREN, 2018).

Feijó (2015) complementa essa ideia ao ressaltar que o cuidado deve ser pertinente para que não haja complicações e o paciente receba alta em bom estado de saúde física e mental.

A Escala de Coma de Glasgow se dá através de um sistema de pontuação de escore, que varia de 3 a 15, sendo obtidos por meio de observações de atividades espontâneas e da aplicação de estímulos verbais e dolorosos, composta por três indicadores, os níveis de abertura ocular, de resposta verbal e de resposta motora (Melo, et al, 2019; Farias, et al, 2021).

Assim, as respostas obtidas na Escala de Glasgow formam a base para a tomada de decisão clinica, ou seja, como se dará o tratamento deste paciente, quais intervenções serão necessárias.

\subsubsection{Elementos da escala}

Como pontuado anteriormente, a escala de coma de Glasgow se dá através de um sistema de pontuação de escore, que varia de 3 a 15, através dos escores de estímulos verbais e dolorosos, como os níveis de abertura ocular, de resposta verbal e de resposta motora (MELO et al, 2019).

Através da tabela, é definido o nível de consciência do paciente acamado, através da resposta ocular, reposta verbal e motora, e assim apontado à pontuação que a pessoa apresenta (Wilberger, 2017). Para que seja avaliada a mesma, são utilizadas as Tabelas 1, 2, 3 e 4 .

Tabela 1 - Níveis da abertura ocular.

\begin{tabular}{|c|c|c|c|c|}
\hline . & 1 & 2 & 3 & 4 \\
\hline Ocular & $\begin{array}{l}\text { Sem abertura dos } \\
\text { olhos }\end{array}$ & $\begin{array}{l}\text { Abre os olhos devido o } \\
\text { estímulo de dor }\end{array}$ & $\begin{array}{l}\text { Abre os olhos } \\
\text { respondendo a um } \\
\text { chamado }\end{array}$ & $\begin{array}{l}\text { Abertura dos olhos } \\
\text { espontâneos }\end{array}$ \\
\hline
\end{tabular}

Fonte: Adaptado de Kouzumi (2000).

Referem-se a quatro níveis:

$4^{\circ}$ Nível - Abertura espontânea;

$3^{\circ}$ Nível - Abertura através de comando verbal;

$2^{\circ}$ Nível - Abertura através de dores; 
$1^{\circ}$ Nível - Sem abertura.

Tabela 2 - Níveis da resposta verbal.

\begin{tabular}{c|c|c|c|c|c}
\hline$\cdot$ & $\mathbf{1}$ & $\mathbf{2}$ & $\mathbf{3}$ & $\mathbf{4}$ & $\mathbf{5}$ \\
\hline Verbal & Emudecido & $\begin{array}{c}\text { Emite sons } \\
\text { incompreensíveis }\end{array}$ & $\begin{array}{c}\text { Pronuncia } \\
\text { palavras } \\
\text { desconexas }\end{array}$ & $\begin{array}{c}\text { Confuso, } \\
\text { desorientado }\end{array}$ & $\begin{array}{c}\text { Orientado, } \\
\text { conversa } \\
\text { normalmente }\end{array}$ \\
\hline
\end{tabular}

Fonte: Adaptado de Kouzumi (2000).

Referem-se a cinco níveis:

$5^{\circ}$ Nível - Orientado, sabe seu nome, idade, local de residência, etc.

$4^{\circ}$ Nível - Confuso, respostas coerentes mas desorientado.

$3^{\circ}$ Nível - Falas aleatórias, palavras inapropriadas.

$2^{\circ}$ Nível - Apresentador som de dores e gemidos. Sem palavras.

$1^{\circ}$ Nível - Sem emissão de sinais sonoros.

Tabela 3 - Níveis da resposta motora.

\begin{tabular}{|c|c|c|c|c|c|c|}
\hline . & 1 & 2 & 3 & 4 & 5 & 6 \\
\hline Motora & $\begin{array}{l}\text { Não se } \\
\text { moviment } \\
\text { a }\end{array}$ & $\begin{array}{c}\text { Extensão a } \\
\text { estímulos } \\
\text { dolorosos } \\
\text { (descerebração) }\end{array}$ & $\begin{array}{c}\text { Flexão anormal } \\
\text { a estímulos } \\
\text { dolorosos } \\
\text { (decorticação) }\end{array}$ & $\begin{array}{c}\text { Flexão } \\
\text { inespecífica } \\
\text { (normal)/ } \\
\text { Reflexo de } \\
\text { retirada a } \\
\text { estímulos } \\
\text { dolorosos }\end{array}$ & $\begin{array}{l}\text { Localiza } \\
\text { estímulos } \\
\text { dolorosos }\end{array}$ & $\begin{array}{l}\text { Obedece a } \\
\text { comandos }\end{array}$ \\
\hline
\end{tabular}

Fonte: Adaptado de Kouzumi (2000).

Referem-se a cinco níveis:

$6^{\circ}$ Nível - Obedece ordens verbais.

$5^{\circ}$ Nível - Apresenta estímulos de dores.

$4^{\circ}$ Nível - Flexão normal.

$3^{\circ}$ Nível - Flexão anormal.

$2^{\circ}$ Nível - Extensão a estímulos dolorosos

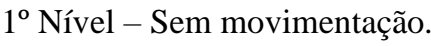

Também contendo a resposta atingida pela reatividade pupilar, através da pupila do mesmo.

Tabela 4 - Reatividade pupilar.

\begin{tabular}{c|c|c|c}
\hline & -2 & -1 & 0 \\
\hline $\begin{array}{c}\text { Reatividade } \\
\text { Pupilar }\end{array}$ & $\begin{array}{c}\text { Sem reação } \\
\text { das pupilas }\end{array}$ & $\begin{array}{c}\text { Apenas uma } \\
\text { (unilateral) }\end{array}$ & $\begin{array}{c}\text { As duas pupilas } \\
\text { (bilateral) }\end{array}$ \\
\hline
\end{tabular}

Fonte: Adaptado de Kouzumi (2000).

Para que seja interpretado da forma correta, contando da pontuação finalizada se indica o estado do paciente, através da escala de coma de Glasgow. 
A pontuação varia de 3 a 15 pontos sendo:

3 pontos: Morte cerebral ou coma profundo, com alta probabilidade de morte e em Síndrome de vigília.

3-8 pontos: Trauma grave

9-12 pontos: Trauma moderado

13-15 pontos: Trauma leve

\subsection{O traumatismo cranioencefálico e os cuidados}

O trauma craniano (TC) é uma lesão física ao tecido cerebral que incapacita as funções do cérebro, permanente ou temporariamente. Seu diagnostico é comprovado por exames e o seu tratamento suporta os auxiliadores respiratórios e a manutenção adequada da ventilação, oxigenação e pressão arterial. (Wilberger, 2017).

Em estimativas coerentes, no Brasil os acidentes de trânsito chegaram a uma marca de 23,4 mortes por mil habitantes, o Brasil está na quarta posição entre os países com mais mortes em acidentes no trânsito no mundo, de acordo com estudo de 2019 da Organização Mundial da Saúde (OMS). O Observatório Nacional de Segurança Viária (Onsv), pontuou que, 1 pessoa morre a cada 15 minutos por causa de acidentes de trânsito no Brasil, e a cada 2 minutos 1 ser humano sofre sequelas por causa de ferimento (Saragiotto, 2020).

Os traumas cranianos não ocorrem apenas em acidentes de trânsitos, mas, também em outros motivos como: acidentes em casa, no ambiente de trabalho ou até mesmo em vias públicas. Segundo alguns estudos, as taxas de TCE são maiores nas crianças (idades entre zero a quatro anos) e em jovens (entre 15 a 24 anos), existe um pico nos idosos (com idade maior que 65 anos) (Ruy, 2011).

O nível de consciência de uma pessoa é um fator essencial para que sejam determinadas as necessidades assistenciais, principalmente daqueles que apresente traumas neurológicos (Cardos, et al, 2017).

Realizar o monitoramento do paciente deve ser efetuado preferencialmente pelo enfermeiro, onde consiste em aplicar a avaliação primária, de acordo com o protocolo o enfermeiro deve garantir permeabilidade das vias aéreas, estabilização da coluna cervical, oferecer oxigênio para uma ventilação adequada, monitorar circulação e o controle da pressão intracraniana e, que pode se tornar alta demais devido a edemas cerebrais e outras condições, condicionante para muitas mortes encefálicas (Souza et al, 2016).

O diagnóstico é suspeitado clinicamente e confirmado por imagens (primariamente TC). Inicialmente, o tratamento consiste em suporte respiratório e manutenção adequada de ventilação, oxigenação e pressão arterial. Possivelmente, a cirurgia será necessária em pacientes com lesões mais graves para a colocação de monitores a fim de medir a elevação da pressão intracraniana, descomprimir o cérebro se a pressão intracraniana estiver aumentada ou remover hematomas intracranianos (Cardos et al, 2017).

\subsection{As mudanças ocorridas na escala de coma de glasgow}

Quarenta anos após a divulgação oficial da ECG, três pesquisadores sendo eles, Teasdale, Paul e Gordon, conduziram outra pesquisa, publicada em 2018, baseada pela experiência dos médicos e enfermeiros mundialmente, e os fatores essenciais da escala, acrescentou a reatividade pupilar dentro dos elementos da mesma. Devido a busca por obter maiores informações sobre o prognóstico no trauma, uma vez que esses estudos demonstraram que há uma precisão maior da observação da escala se adicionada à reatividade pupilar.

Essas alterações feitas na escala, influenciaram gradativamente no esclarecimento das informações referente a pontuação dentro dos 4 (quatro) testes das respostas do paciente (verbal, motora, ocular e a reação da pupila). 
Dentro do que diz respeito à estrutura da escala, a modificação ocorrida foi à prioridade da pontuação individual (tornando-a com maior ênfase), melhor do que a pontuação total. As nomenclaturas foram revisadas, para que haja mais precisão na efetuação do registro quanto a forma do estimulo. Um exemplo mesmo é quanto ao termo "dor", pois havia bastante questionamento sobre a necessidade da avaliação em nos pacientes em coma. Presente no aspecto de resposta motora houve a divisão dos termos flexões "normais" e "anormais", para facilitar a identificação da pontuação. O escore do paciente quando tratado a reação pupilar, é uma questão observada para que seja tomada as ações mais rápidas, evitando assim consequências radicais, logo deve ser analisada após a contagem tradicional, sendo subtraída da contagem geral da escala, tendo a situação presente do paciente.

A Tabela 5 faz um comparativo de como era as respostas e como deve ser observado no momento.

Tabela 5 - Escala anterior x escala nova - sobre a ECG.

\begin{tabular}{|c|c|c|c|}
\hline \multicolumn{4}{|c|}{ RESPOSTA MOTORA } \\
\hline \multicolumn{2}{|c|}{ Escala anterior } & \multicolumn{2}{|c|}{ Escala nova } \\
\hline Variáveis & Pontuação & Variáveis & Pontuação \\
\hline Obedece a comandos & 6 & A ordens & $\theta$ \\
\hline Localiza dor & 5 & Localizadora & 5 \\
\hline Movimento de retirada & 4 & Flexão normal & 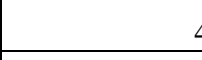 \\
\hline Decorticação & 3 & Flexão anormal & 3 \\
\hline Descerebração & 2 & Extensão & 2 \\
\hline \multirow[t]{2}{*}{ Nenhuma } & 1 & Ausente & 1 \\
\hline & & Não testável & NT \\
\hline \multicolumn{4}{|c|}{ RESPOSTA VERBAL } \\
\hline \multicolumn{2}{|c|}{ Escala anterior } & \multicolumn{2}{|c|}{ Escala nova } \\
\hline Variáveis & Pontuação & Variáveis & Pontuação \\
\hline Orientada & 5 & Orientada & $s$ \\
\hline Confusa & 4 & Confusa & 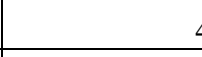 \\
\hline Palavras inapropriadas & 3 & Palavras & 3 \\
\hline Palavras incompreensíveis & 2 & Sons & 2 \\
\hline \multirow[t]{2}{*}{ Nenhuma } & 1 & Ausente & 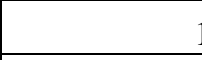 \\
\hline & & Não testável & NT \\
\hline \multicolumn{4}{|c|}{ ABERTURA OCULAR } \\
\hline \multicolumn{2}{|c|}{ Escala anterior } & \multicolumn{2}{|c|}{ Escala nova } \\
\hline Variáveis & Pontuação & Variáveis & Pontuação \\
\hline Espontânea & 4 & Espontânea & 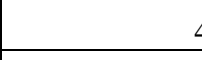 \\
\hline A voz & 3 & Ao som & 3 \\
\hline A dor & 2 & A pressão & 2 \\
\hline \multirow[t]{2}{*}{ Nenhuma } & 1 & Ausente & 1 \\
\hline & & Não testável & NT \\
\hline
\end{tabular}

Fonte: Adaptado de Brennan, Murray e Teasdale (2018). 
Essa nova atualização os pesquisadores adicionaram mais um critério assertivo para a definição da gravidade de um trauma, sendo a reatividade popular, tratada como a nomenclatura define, as reações das duas pupilas e sendo subtraída pelo valor da pontuação geral (somatório entre a resposta motora e a verbal e a abertura ocular).

Tabela 6 - Reatividade pupilar.

\begin{tabular}{c|c|c|c}
\hline & -2 & -1 & 0 \\
\hline $\begin{array}{c}\text { Reatividade } \\
\text { Pupilar }\end{array}$ & $\begin{array}{c}\text { Não há } \\
\text { reação das } \\
\text { pupilas }\end{array}$ & $\begin{array}{c}\text { Unilateral } \\
\text { (apenas } \\
\text { uma } \\
\text { reagem) }\end{array}$ & $\begin{array}{c}\text { Bilateral } \\
\text { (as duas } \\
\text { reagem) }\end{array}$ \\
\hline
\end{tabular}

Fonte: Adaptado de Brennan, Murray e Teasdale (2018).

Adicionado a reatividade pupilar (RA), houve mudanças na variação da escala anteriormente estava entre 3 e 15 pontos, com o novo critério da RA, obtêm-se um novo valor variável entre 1 e 15.

\subsubsection{Fórmula da pontuação da ECG}

O cálculo (pontuação) da escala era feito o somatório de cada critério usando a abreviação e a resposta, tendo a resposta da ECG.

$$
\begin{array}{lr}
E C G=R M+R V+A O & \mathbf{E C G}=\text { Escala de Coma de Glasgow } \\
E C G=3+4+2 & \mathbf{R M}=\text { Resposta Motora } \\
E C G=9 & \mathbf{R V}=\text { Resposta Verbal } \\
& \mathbf{A O}=\text { Abertura Ocula }
\end{array}
$$

Logo, com a atualização da ECG, não será mais apenas a pontuação e sim a pontuação de cada critério junto com a abreviação, na somatória. Para que haja a quantificação do resultado, posterior a avaliação, deve ser escrito a letra correspondente a cada critério e a pontuação após a letra e, por fim, somará todos os números pertencentes a formula e terá o resultado.

$$
\begin{array}{lc}
E C G=0 n V n M n-R P & \mathbf{E C G}=\text { Escala de Coma de Glasgow } \\
E C G=03 V 4 M 2-0 & \mathbf{M}=\text { Resposta Motora } \\
E C G=9 & \mathbf{V}=\text { Resposta Verbal } \\
& \mathbf{O}=\text { Abertura Ocular } \\
& \mathbf{R P}=\text { Reatividade pupilar }
\end{array}
$$

A saúde e os instrumentos, vão se habilitando em tecnologias e se atualizando dentro da século vivido, as mudanças são significativas e propõe qualidade no atendimento prestado diariamente por todo corpo técnico de enfermagem, confortando o paciente no ambiente seguro, além de aprimorar os profissionais. 
Uma questão, que se deve ter atenção é que nem todos os pacientes são susceptíveis a avaliação da forma como a antiga escala de glasgow propõe. Exemplo são pacientes amputados que não obrigatoriamente terão uma avaliação autentica da resposta motora, pacientes que tenham surdez não serão atenderão a comandos verbais e pacientes com algum quadro de enfraquecimento da fala (afasia) possivelmente não conseguirão exalar respostas verbais.

Sendo consideradas essas necessidades individuais de alguns pacientes, nesta nova atualização, poderá marcá-los como "não testável" (nt).

\section{Metodologia}

\subsection{Classificação da pesquisa}

O presente artigo se classifica como descritivo de natureza básico visto seu caráter de descrição de um fenômeno com suas variáveis. Dessa forma, Gil (2017) discorre que essa pesquisa visa descrever as características de um fenômeno, a fim de compreender todos os seus aspectos, tomando como base o objetivo de analisar as produções acadêmicas acerca da temática Aplicação da Escala de Coma de Glasgow.

Quanto aos procedimentos técnicos essa pesquisa se classifica como uma revisão sistemática da bibliografia com foco na técnica do estudo bibliométrico. A bibliométria é uma técnica de pesquisa utilizada para medir e interpretar os índices da produção cientifica, o método quantitativo e estatístico de determinado objeto de estudo (Araújo, 2006).

A utilização desta técnica permitiu quantificar as principias aplicações da Escala de Coma de Glasgow, possibilitando uma discussão acerca dos diferentes resultados dos estudos. Para alcançar os objetivos propostos foi utilizado uma pesquisa de abordagem quantitativa visto que seu objetivo é mensurar as aplicações da temática, fazendo uso da estatística para descrever o fenômeno, suas variáveis e as relações entre as aplicações (Miguel, 2012).

Os instrumentos de pesquisa consistem, portanto em estatística e analise critica, onde as informações coletadas serão dispostas em tabelas e gráficos, com o auxilio do Software Microsoft Excel, os quais serão seguidos por inferências e discussões acerca dos dados apresentados, para interpretar as aplicações do fenômeno objeto de estudo.

A princípio foi-se realizado uma pesquisa bibliográfica acerca da temática, a fim de levantar as referencias do tema proposto, a posteriori serão realizados uma metanálise a partir do estudo bibliométrico para levantar a discussão da temática.

\subsection{Procedimentos da Pesquisa}

Essa pesquisa se deu com base em alguns procedimentos, a partir da pesquisa dos artigos com base em palavras chaves, como escala de Coma de Glasgow, aplicação da escala de Coma de Glasgow. Os artigos foram selecionados com base em critérios de inclusão como ano de publicação, qualis do periódico, bases de dados, área de atuação, titulação e instituição de vínculo, local de origem de estudo, idioma, metodologia, nível de evidência, quantitativo de referências e principais enfoques.

Nesse contexto, os artigos foram analisados por meio da técnica bibliométrica (metanálise) onde foram feitos gráficos e tabelas acercados resultados, para o levante da discussão. A partir dessas etapas foram detalhados os trabalhos científicos selecionados e se tabulados os dados com o intuito de descrever a partir da estatística os principais resultados.

\subsection{Analise dos dados}

Com base nos critérios de inclusão e exclusão, detalhados nos procedimentos da pesquisa foram selecionados nove artigos, dos dezessete encontrados. Os artigos selecionados foram publicados entre 2011 e 2021, tem como enfoque a escala de glasgow, a percepção dos enfermeiros, e as implicações para pacientes com traumatismo cranioencefálico (tce).

A tabela abaixo contempla os artigos selecionados e descreve suas características. 
Tabela 7 - Perfil e características dos artigos selecionados.

\begin{tabular}{|c|c|c|c|c|}
\hline TÍTULO & AUTORES / ANO & OBJETIVO & METODOLOGIA & RESULTADOS \\
\hline $\begin{array}{c}\text { Correlação entre a escala de coma de } \\
\text { Glasgow e os achados de imagem de } \\
\text { tomografia computadorizada em } \\
\text { pacientes vítimas de traumatismo } \\
\text { cranioencefálico }\end{array}$ & $\begin{array}{l}\text { MORGADO e ROSSI } \\
\text { (2011) }\end{array}$ & $\begin{array}{l}\text { Determinar a correlação da } \\
\text { escala de coma de Glasgow, } \\
\text { fatores causais e de risco, } \\
\text { idade, sexo e intubação } \\
\text { orotraqueal com os achados } \\
\text { tomográficos em pacientes } \\
\text { com traumatismo } \\
\text { cranioencefálico }\end{array}$ & $\begin{array}{l}\text { Estudo transversal com } \\
\text { pacientes a partir da escala } \\
\text { de coma de Glasgow e de } \\
\text { exame tomográfico }\end{array}$ & $\begin{array}{l}\text { A partir dos estudos foram analisados as principias } \\
\text { causas, identificando atropelamentos, quedas, } \\
\text { acidente automobilístico e agressão. Além disso, } \\
\text { foram observadas alterações tomográficas } \\
\text { (hematoma subgaleal, fraturas ósseas da calota } \\
\text { craniana, hemorragia subaracnoidea, contusão } \\
\text { cerebral, coleção sanguínea extra-axial, edema } \\
\text { cerebral difuso) em } 79,42 \% \text { dos pacientes. }\end{array}$ \\
\hline $\begin{array}{l}\text { Escala de coma de Glasgow e qualidade } \\
\text { de vida pós-trauma cranioencefálico }\end{array}$ & $\begin{array}{l}\text { SETTERVALL; } \\
\text { SOUSA (2011) }\end{array}$ & $\begin{array}{l}\text { Avaliar o desempenho de } \\
\text { diferentes escores da Escala } \\
\text { de Coma de Glasgow (ECGl) } \\
\text { observados nas primeiras } 72 \\
\text { horas pós trauma perante a } \\
\text { qualidade de vida e mudança } \\
\text { percebida do estado de saúde, } \\
\text { após um ano do evento } \\
\text { traumático }\end{array}$ & $\begin{array}{c}\text { Estudo de abordagem } \\
\text { quantitativa, observacional, } \\
\text { longitudinal, descritivo e } \\
\text { correlacional com vítimas } \\
\text { de trauma cranioencefálico } \\
\text { contuso (TCEC) avaliadas, } \\
\text { diariamente durante a } \\
\text { internação hospitalar, e após } \\
\text { um ano por meio do } \\
\text { Medical OutcomeStudy 36- } \\
\text { item Short Form Health } \\
\text { Survey (SF-36) } \\
\end{array}$ & $\begin{array}{l}\text { Sob as curvas RecieverOperatorCharacteristics dos } \\
\text { valores da ECGl referentes à mudança percebida do } \\
\text { estado de saúde não apresentaram diferença } \\
\text { significativa e variaram de } 0,63 \text { a } 0,71 \text {. Correlação, } \\
\text { estatisticamente significante, porém fraca, foi } \\
\text { observada entre os escores da ECGl e alguns dos } \\
\text { domínios do SF-36. Verificou-se que os diferentes } \\
\text { valores da ECGl apresentaram limitações para que } \\
\text { fossem aplicados na prática clínica para estimar as } \\
\text { consequências do TCEC a longo prazo }\end{array}$ \\
\hline $\begin{array}{l}\text { Avaliação do conhecimento de } \\
\text { enfermeiros sobre a escala de coma de } \\
\text { Glasgow em um hospital universitário }\end{array}$ & SANTOS et al (2016) & $\begin{array}{l}\text { Avaliar o conhecimento de } \\
\text { enfermeiros de unidades } \\
\text { críticas, serviços de } \\
\text { emergência e unidades de } \\
\text { terapia intensiva em relação à } \\
\text { escala de coma de Glasgow }\end{array}$ & $\begin{array}{l}\text { Estudo transversal e } \\
\text { analítico com } 127 \\
\text { enfermeiros de unidades } \\
\text { críticas de um hospital } \\
\text { universitário. Utilizou-se } \\
\text { entrevista estruturada com } \\
12 \text { questões que avaliaram } \\
\text { conhecimento sobre a } \\
\text { escala. }\end{array}$ & $\begin{array}{c}\text { Foi possível identificar que enfermeiros com maior } \\
\text { tempo de atuação demonstraram maior } \\
\text { conhecimento acerca da escala, mesmo nesse } \\
\text { sentido houveram grande número de erros, os } \\
\text { enfermeiros das unidades de terapia intensiva se } \\
\text { demonstraram com maior conhecimento, ou seja, } \\
\text { tempo de formação, experiência e trabalho na } \\
\text { unidade interferiu no conhecimento de enfermeiros } \\
\text { sobre a escala, evidenciando necessidade de } \\
\text { capacitação } \\
\end{array}$ \\
\hline $\begin{array}{c}\text { Aplicação de um programa de } \\
\text { estimulação multissensorial a doentes } \\
\text { com alterações severas do estado de } \\
\text { consciência }\end{array}$ & $\begin{array}{l}\text { RODRIGUES e } \\
\text { VARANDA (2019) }\end{array}$ & $\begin{array}{l}\text { Promover a melhoria do } \\
\text { estado de consciência destes } \\
\text { doentes }\end{array}$ & $\begin{array}{l}\text { Aplicação de um programa } \\
\text { de Estimulação } \\
\text { Multissensorial (PEM) }\end{array}$ & $\begin{array}{l}\text { Dos resultados destacamos: mais de metade dos } \\
\text { doentes evoluíram no estado de consciência, 63,64\% } \\
\text { melhoraram o Score na ECG e 54, 55\% progrediram } \\
\text { no nível LCFS; os que tiveram a família envolvida } \\
\text { evoluíram seis vezes mais em média na ECG. } \\
\text { Concluímos que com a aplicação do nosso PEM } \\
\text { poderemos contribuir para a melhoria do estado de } \\
\text { consciência da maioria destes doentes }\end{array}$ \\
\hline
\end{tabular}


Research, Society and Development, v. 10, n. 14, e48101421643, 2021

(CC BY 4.0) | ISSN 2525-3409 | DOI: http://dx.doi.org/10.33448/rsd-v10i14.21643

\begin{tabular}{|c|c|c|c|c|}
\hline $\begin{array}{l}\text { Utilização da escala de Glasgow para } \\
\text { detecção precoce de complicações }\end{array}$ & MELO et al (2019) & $\begin{array}{l}\text { Avaliar a utilização da escala } \\
\text { de coma de Glasgow para } \\
\text { detectação precoce de } \\
\text { complicações neurológicas }\end{array}$ & $\begin{array}{l}\text { Revisão integrativa da } \\
\text { literatura utilizando base de } \\
\text { dados da Biblioteca Virtual } \\
\text { de Saúde (BVS) e SCIELO, } \\
\text { LILACS de } 2005 \text { a } 2019\end{array}$ & $\begin{array}{l}\text { A aplicação da escala de Glasgow se dá através de } \\
\text { uma ficha de avaliação neurológica empregada } \\
\text { conforme a gravidade do paciente, onde cada } \\
\text { indicador deve ser avaliado independente dos outros } \\
\text { É essencial salientar que a pontuação dever ser dada } \\
\text { para a melhor resposta do paciente, em cada } \\
\text { indicador, acrescida da pontuação total, ou seja, a } \\
\text { somatória dos três indicadores. }\end{array}$ \\
\hline $\begin{array}{c}\text { As entrelinhas da literatura no tocante ao } \\
\text { uso da escala de coma de Glasgow por } \\
\text { Enfermeiros }\end{array}$ & SOUZA et al (2020) & $\begin{array}{l}\text { Sistematizar o conhecimento } \\
\text { produzido acerca do uso da } \\
\text { ECG por Enfermeiros }\end{array}$ & $\begin{array}{l}\text { Revisão Integrativa da } \\
\text { Literatura }\end{array}$ & $\begin{array}{c}\text { A utilização da ECG faz parte da rotina dos } \\
\text { profissionais de saúde principalmente em unidades } \\
\text { de terapia intensiva onde os pacientes necessitam de } \\
\text { uma avaliação e monitorização mais criteriosa, } \\
\text { sendo uma ferramenta auxiliar no desenvolvimento } \\
\text { do trabalho do enfermeiro, na tomada de decisões } \\
\text { apropriadas e da interpretação dos resultados com } \\
\text { mais eficiência e segurança } \\
\end{array}$ \\
\hline $\begin{array}{l}\text { Conhecimento de enfermeiros na } \\
\text { abordagem à vítima de traumatismo } \\
\text { cranioencefálico }\end{array}$ & $\begin{array}{l}\text { REZER; PEREIRA; } \\
\text { FAUSTINO (2020) }\end{array}$ & $\begin{array}{l}\text { Verificar o conhecimento de } \\
\text { enfermeiros sobre o } \\
\text { traumatismo cranioencefálico } \\
\text { e a Escala de Coma de } \\
\text { Glasgow }\end{array}$ & $\begin{array}{c}\text { Estudo exploratório } \\
\text { realizado por meio de } \\
\text { entrevistas com enfermeiros } \\
\text { atuantes em serviços } \\
\text { hospitalares de urgência e } \\
\text { emergência. }\end{array}$ & $\begin{array}{l}\text { O estudo aponta a importância dos enfermeiros se } \\
\text { manterem atualizados no atendimento às vítimas de } \\
\text { traumatismo cranioencefálico, além da necessidade } \\
\text { de educação permanente e aperfeiçoamento da } \\
\text { equipe, para melhoria do atendimento e segurança } \\
\text { do paciente, visto que } 60 \% \text { dos enfermeiros } \\
\text { entrevistados identificaram barreiras no atendimento } \\
\text { às vítimas de traumatismo crânio encefálico. }\end{array}$ \\
\hline $\begin{array}{c}\text { Processo de Enfermagem Informatizado } \\
\text { Apoiado por Sistema Especialista na } \\
\text { Aplicação das Escalas de Braden e de } \\
\text { Glasgow }\end{array}$ & FARIAS et al (2020) & $\begin{array}{l}\text { Apresentar a implementação, } \\
\text { em hospital público de médio } \\
\text { porte, do processo de } \\
\text { enfermagem informatizado } \\
\text { apoiado por SE na aplicação } \\
\text { das escalas de Braden e de } \\
\text { Glasgow } \\
\end{array}$ & $\begin{array}{l}\text { Bibliográfica aplicada e } \\
\text { experimental. }\end{array}$ & $\begin{array}{c}\text { Tanto o processo de enfermagem informatizado } \\
\text { quanto o SE foram implementados e os resultados } \\
\text { parciais obtidos são positivos, porém necessitam de } \\
\text { maior tempo de análise e avaliação. }\end{array}$ \\
\hline $\begin{array}{l}\text { Avaliação do conhecimento de } \\
\text { estudantes da área da saúde sobre a } \\
\text { Escala de Coma de Glasgow em uma } \\
\text { Universidade de Minas Gerais }\end{array}$ & $\begin{array}{l}\text { COUTO; SILVA; } \\
\text { CARDOSO (2021) }\end{array}$ & $\begin{array}{c}\text { Analisar e avaliar o } \\
\text { conhecimento de estudantes } \\
\text { da área da saúde sobre a } \\
\text { função e aplicabilidade da } \\
\text { Escala de Coma de Glasgow } \\
\text { (ECG) }\end{array}$ & $\begin{array}{l}\text { Estudo de caso, aplicação } \\
\text { de questionário aos } \\
\text { acadêmicos do último ano } \\
\text { dos cursos de Medicina, } \\
\text { Enfermagem, Odontologia e } \\
\text { Farmácia da Universidade } \\
\text { Federal de Alfenas }\end{array}$ & $\begin{array}{l}\text { Os resultados revelam que os futuros médicos e } \\
\text { enfermeiros apresentam maior conhecimento dos } \\
\text { parâmetros e aplicação da ECG quando comparado } \\
\text { aos demais estudantes avaliados, isso é um } \\
\text { empecilho para excelência do cuidado em saúde, } \\
\text { visto que as equipes multiprofissionais são de } \\
\text { extrema importância para o cuidado, e seus } \\
\text { membros necessitam de um conhecimento ampliado } \\
\text { sobre ECG para fornecer ao paciente um } \\
\text { monitoramento seriado e evitar problemas de } \\
\text { comunicação entre a equipe }\end{array}$ \\
\hline
\end{tabular}

Fonte: Autores (2021). 
A partir dos artigos selecionados foi realizado a tabulação dos dados com o intuito de descrever a partir da estatística os principais resultados encontrados com as aplicações das metodologias descritas na Tabela 1 acima, e realizar uma discussão acerca destes dados.

\section{Resultados e Discussão}

A partir da descrição dos nove artigos selecionados, foi realizada a distinção entre os tipos de estudos, pode-se notar que apenas dois são revisões de integrativas da literatura, o que corresponde a 22, $22 \%$ dos artigos selecionados, dois são estudos transversais o que corresponde mais $22,22 \%$, os outros cinco artigos são aplicações, estudos de caso e relatos descritivos, o que corresponde a 55,56\% dos artigos selecionados.

O gráfico abaixo demonstra essa distinção dos artigos em porcentagens.

Gráfico 1 - Distinção metodológica dos artigos selecionados para analise.

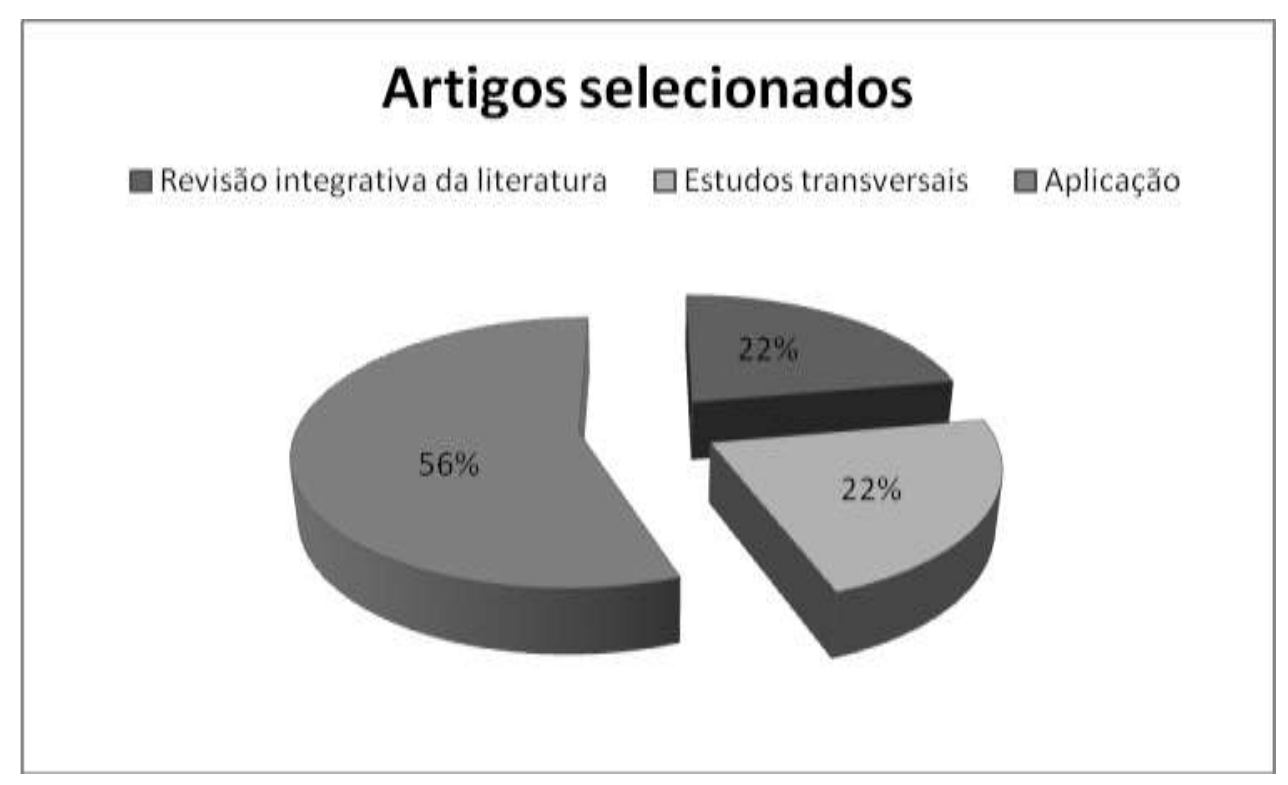

Fonte: Autores (2021).

O artigo denominado “utilização da escala de glasgow para detecção precoce de complicações” de Melo et al (2019) buscou através da bases de dados biblioteca virtual de saúde (bvs) e scielo, lilacs artigos publicados entre 2005 e 2019 a fim de avaliar a utilização da escala de coma de glasgow para detectação precoce de complicações neurológicas.

Melo et al (2019) identifica a partir de uma seleção e analise de 6 artigos que a escala de glasgow é muito utilizada pelos profissionais da saúde para facilitar no reconhecimento dos sinais de alerta que estão presente em pacientes em situação grave, assim otimizando a intervenção precoce, diminuindo ou evitando a lesão secundária neurológica.

Já Souza et al no artigo "as entrelinhas da literatura no tocante ao uso da escala de coma de glasgow por enfermeiros", identificaram com base na analise de 10 artigos a escala de coma de glasgow (ecg) é mais utilizada como ferramenta de avaliação de nível de consciência. O artigo dos autores supracitados apresentam estudos de níveis exploratórios, descritivos, transversais e observacionais, que em suas diferentes abordagens trataram a ecg como uma ferramenta de avaliação e investigação clinica do estado do paciente.

Nesse contexto, nos artigos que tratam de metodologias de revisão de literatura, é possível se entende e compreende a importância do profissional da enfermagem no que condiz a avaliação, visto a necessidadede observação atenta e criteriosa dos 
níveis que compõem a quantificação da escala, itens como nível de consciência, dilatação da pupila, resposta motora, dentre outros.

Os artigos que trabalham com metodologia de estudo transversal, são os artigos de Santos et al (2016) denominado “Avaliação do conhecimento de enfermeiros sobre a escala de coma de Glasgow em um hospital universitário" e o estudo de Morgado e Rossi (2011) denominado "Correlação entre a escala de coma de Glasgow e os achados de imagem de tomografia computadorizada em pacientes vítimas de traumatismo cranioencefálico".

O primeiro estudo, de Santos et al (2016) trabalha com uma amostra de 127 enfermeiros, utilizando uma entrevista com 12 perguntas, a fim de avaliar o conhecimento destes enfermeiros acerca da escala. Dos 127 entrevistados, 99\% sabiam para que servia a escala de coma, 98\% tinham conhecimento acerca dos indicadores do ECG, e em um contexto geral $80 \%$ dos entrevistados possuíam conhecimento da aplicação e utilização da ECG.

No estudo de Morgado e Rossi (2011) por outro lado, o estudo foi realizado com pacientes, contendo uma amostra de102 indivíduos. O objetivo deste estudo era determinar a correlação da escala de coma de Glasgow, fatores causais e de risco, idade, sexo e intubação orotraqueal com os achados tomográficos em pacientes com traumatismo cranioencefálico.

A partir da analise de todos estes fatores os autores puderam perceber que a idade média dos pacientes foi de 37,77 \pm 18,69 anos, com predomínio do sexo masculino $(80,4 \%)$. As causas foram: acidente automobilístico $(52,9 \%)$, queda de outro nível (20,6\%), atropelamento (10,8\%), queda ao solo ou do mesmo nível (7,8\%) e agressão (6,9\%).

De uma forma geral os dois artigos analisados abordam os fatores de risco para os pacientes, demonstrando que a pontuação do ECG contribui para o prognostico e para o tratamento especializado do paciente.

Os artigos de aplicações, estudos de caso e relatos descritivos que compõem os 55,56\% dos artigos analisados, trabalham percepções diferentes. O primeiro estudo desta seqüência de artigos é o texto de Settervall e Sousa (2011) denominado "Escala de coma de Glasgow e qualidade de vida pós-trauma cranioencefálico", trata-se de um artigo com abordagem qualitativa e estudo observacional.

A partir dessa metodologia as autoras conseguiram avaliar o desempenho dos diferentes escores da Escala de Coma de Glasgow (ECGI) nas primeiras 72 horas do pós-trauma, evidenciando assim, que da amostragem de 277 pacientes, $43 \%$ apresentavam Traumatismo cranioencefálico (TCEC) leve, 16,2\% moderado e 40,8\% TCEC grave, identificado conforme escores de ECGl após a reanimação inicial no hospital.

É importante esse levantamento de discussão acerca do desempenho no que concerne a qualidade de vida do paciente, dessa forma, os autores supracitados identificaram que os resultados obtidos pela ECG podem servir com prognostico para determinar resultados a longo prazo em relação a qualidade de vida do paciente.

O artigo denominado "Aplicação de um programa de estimulação multissensorial a doentes com alterações severas do estado de consciência” de Rodrigues e Varanda (2019) se inter-relaciona com o estudo pontuado anteriormente de Settervall e Sousa (2011), visto a busca da qualidade e melhoria continua para o paciente por meio da pratica de enfermagem e aplicação da ECG.

Este estudo realizou a Promoção da melhoria do estado de consciência destes doentes através da aplicação de um programa de Estimulação Multissensorial (PEM) e obteve como resultado que a aplicação deste programa auxilia na evolução do paciente, através da aplicação do ECG é possível analisar os níveis de consciência do indivíduo, além de contribuir para diagnósticos tais quais o AVC hemorrágico e o TCE.

O texto "Conhecimento de enfermeiros na abordagem à vítima de traumatismo cranioencefálico" de Rezer, Pereira e Faustino (2020) compreendeu em seu contexto geral que dos 20 enfermeiros entrevistados $90 \%$ dos já atenderam pacientes com traumatismo cranioencefálico e $95 \%$ se dizem preparados para esse atendimento. Grande parte dos enfermeiros diz que existem barreiras no atendimento às vítimas de traumatismo crânio encefálico, o que corresponde a $60 \%$ dos entrevistados, 
como equipe despreparada um total de $20 \%$ e falta de estrutura $40 \%$. Em relação à classificação do traumatismo, $70 \%$ acertaram quanto ao leve, $95 \%$ ao moderado e $75 \%$ ao grave.

Esse estudo demonstra mais uma vez a ideia que vem sendo discutida até aqui, de que os enfermeiros são de suma importância para este processo, e que estes devem sempre se manterem atualizados para realizar o atendimento às vítimas de traumatismo cranioencefálico.

Em seu texto "Processo de Enfermagem Informatizado Apoiado por Sistema Especialista na Aplicação das Escalas de Braden e de Glasgow", Farias et al (2020) apresentam a implementação, em hospital público de médio porte, um processo de enfermagem informatizado apoiado por Sistema especialista (SE) na aplicação das escalas de Braden e de Glasgow.

Esse estudo demonstrou a positividade e viabilidade da aplicação do sistema, havendo agora uma padronização nos serviços ofertados pelo hospital, e nas praticasdo enfermeiros, no entanto, os autores ressaltam que os resultados parciais obtidos são positivos, porém necessitam de maior tempo de análise e avaliação.

O ultimo artigo denominado “Avaliação do conhecimento de estudantes da área da saúde sobre a Escala de Coma de Glasgow em uma Universidade de Minas Gerais" de Couto, Silva e Cardoso (2021) analisou e avaliou o conhecimento dos estudantes sobre a aplicabilidade da ECG.

Foi elaborado um questionário com cinco perguntas para avaliar o conhecimento dos estudantes, os resultados do estudo nos revelam que os estudantes de medicina e enfermagem apresentam maior conhecimento dos parâmetros e aplicação da ECG quando comparado aos demais estudantes avaliados, dos cursos de farmácia e odontologia.

Esses resultados demonstram um problema acerca da formação destes estudantes, e causa preocupação no sentido da analise dos futuros profissionais, visto que as equipes multiprofissionais são de extrema importância para o cuidado do paciente, e o conhecimento ampliado sobre ECG pode fornecer ao paciente um monitoramento seriado e evitar problemas de comunicação entre a equipe.

Assim, a partir da analise dos artigos aqui discutidos podemos observar os resultados acerca da utilização da ECG para tratamento e diagnóstico, e o conhecimento dos enfermeiros acerca desta metodologia. A Tabela 8 abaixo demonstra essa linha de resultados.

Tabela 8 - Resultado acerca do conhecimento da enfermagem e utilização da ECG.

\begin{tabular}{c|c|c}
\hline $\mathbf{N}^{\mathbf{0}}$ & ARTIGO & RESULTADO \\
\hline 1 & MORGADO e ROSSI (2011) & Resultados Satisfatórios \\
\hline 2 & SETTERVALL; SOUSA (2011) & Resultados Satisfatórios \\
\hline 3 & SANTOS et al (2016) & Resultados Satisfatórios \\
\hline 4 & RODRIGUES e VARANDA (2019) & Resultados Satisfatórios \\
\hline 5 & MELO et al (2019) & Resultados Satiórios \\
\hline 6 & SOUZA et al $(2020)$ & Resultados \\
\hline 7 & REZER; PEREIRA; FAUSTINórios \\
\hline 8 & FARIAS et al (2020) & Resultados Intermediários \\
\hline 9 & COUTO; SILVA; CARDOSO (2021) & Resultados Insatisfatórios \\
\hline
\end{tabular}

Fonte: Autores (2021).

Abaixo o gráfico demonstra esses resultados em porcentagem. 
Gráfico 2 - Porcentagem dos resultados dos artigos analisados.

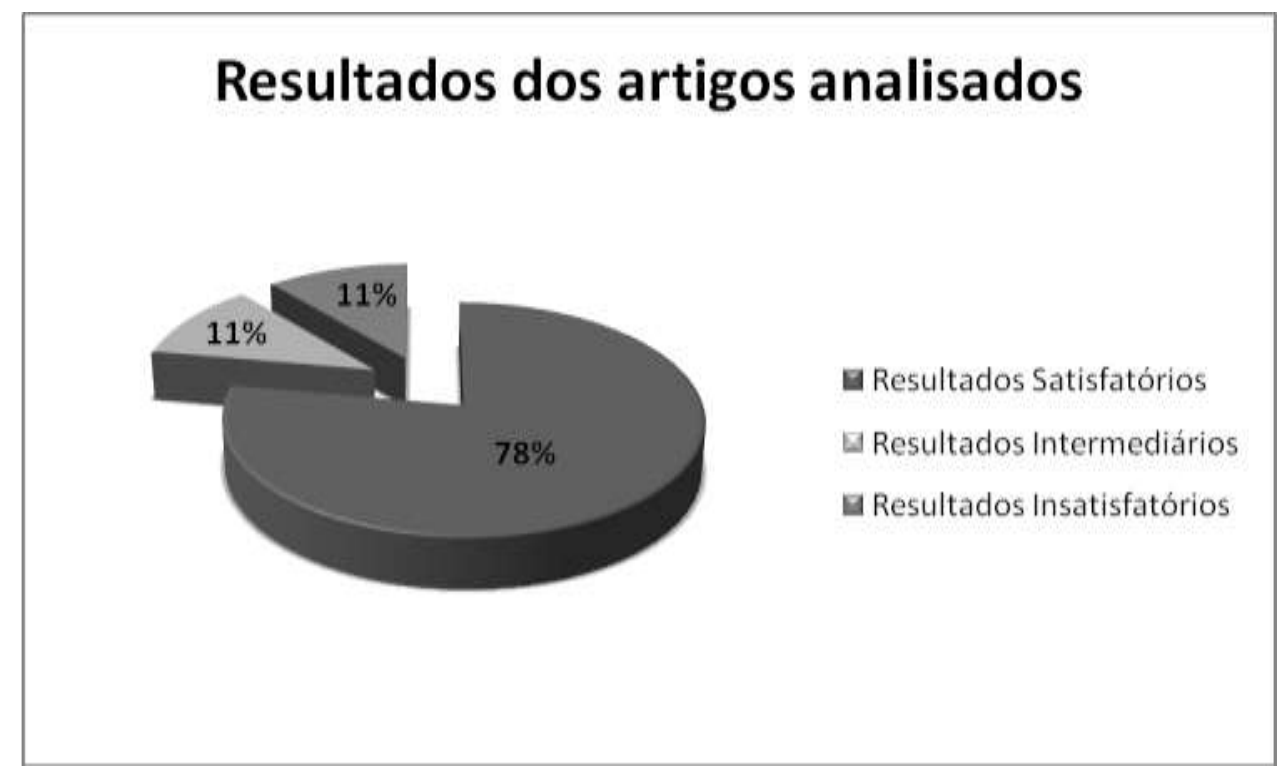

Fonte: Autores (2021).

Nesse sentido, numa analise percentual pode-se notar que a ecg contribui significativamente para a rotina dos profissionais de enfermagem, principalmente para os que atuam em unidades de terapia intensiva, sendo uma excelente ferramenta para o desenvolvimento do trabalho deste profissional, para o auxilio na tomada assertiva de decisões e na interpretação dos dados com eficiência.

O cuidado prestado pelo enfermeiro requer conhecimento e múltiplas capacidades deste profissional, que quando aliado a experiência profissional, determina o alcance da boa assistência de enfermagem, assim como demonstrado em $78 \%$ dos estudos aqui pontuados.

Assim, a literatura confirma que ainda há discrepância no conhecimento e na aplicação da ECG visto que 11,11\% dos resultados foram insatisfatórios e/ou intermediários, isso contribui para o levantamento de que são necessários outros estudos que aprofundem na verificação da eficácia em relação ao aprendizado teórico e pratico nas Instituições de ensino superior acerca da Escala de Coma de Glasgow.

\section{Conclusão}

A partir deste estudo foi possível identificar e alcançar o seu objetivo principal, que era realizar uma analise bibliométrica de diferentes estudos acerca da Aplicação da Escala de Coma de Glasgow, onde se pode identificar que esta é muito empregada hoje para identificar disfunções neurológicas, acompanhar a evolução do nível de consciência, predizer prognósticos e padronizar a linguagem entre os profissionais de saúde.

Além do mais foi possível identificar a partir desta analise bibliométrica que a ECG compõe o dia a dia dos profissionais de saúde, auxiliando no desenvolvimento do trabalho, na avaliação e monitorização e tomada assertiva de decisão.

Nesse sentido compreende-se a partir dos artigos aqui analisados e discutidos que o profissional de enfermagem deve sempre buscar se manter atualizado, para realizar o atendimento às vítimas de traumatismo cranioencefálico que necessitam da utilização da Escala de Coma de Glasgow.

Compreende-se ainda que a literatura acerca dessa temática abrange em sua maioria aplicação da escala e analise de conhecimento dos profissionais de enfermagem acerca da escala, o que nos possibilitou identificar a partir da seleção de 9 
artigos que 78\% dos estudos aqui pontuados alcançaram objetivos positivos demonstrado o conhecimento dos profissionais e as vantagens de aplicação da ECG.

Já 11,11\% dos resultados foram insatisfatórios e/ou intermediários, ou seja, um somatório de 22, 22\% dos artigos pontuados obteve resultados negativos, o que nos leva ao entendimento de que em alguma aplicação o conhecimento da ECG se faz insatisfatório.

Isso, nos leva ao entendimento de se fazem necessários outros estudos que aprofundem a verificação da eficácia em relação ao aprendizado teórico e prático nas Instituições de ensino superior acerca da Escala de Coma de Glasgow, o que se torna uma sugestão para futuros trabalhos.

\section{Referências}

Araújo, C. A. (2006). Bibliométria: evolução histórica e questões atuais. Em Questão, 12 (1), 11-32.

Brennan, P. M., Murray, G. D. \& Teasdale, G. M. (2018). Simplifyingthe use ofprognosticinformation in traumaticbraininjury: part 1: thegcs-pupils score: anextended index ofclinicalseverity. JournalOfNeurosurgery.

Cardos, A. V. O. (2017). Uso da Escala de Coma de Glasgow para avaliação do nível de consciência de pacientes com traumatismo crânio encefálico. REAS, Revista Eletrônica Acervo Saúde, (Sup. 5), 249-55. https://acervomais.com.br/index.php/saude/article/view/7842/4817

Couto, D. S; Silva, N. B \& Cardoso, E. J. R. (2021). Avaliação do conhecimento de estudantes da área da saúde sobre a Escala de Coma de Glasgow em uma Universidade de Minas Gerais. Research, Society and Development, 10 (9).

Farias, E. B. P; et al. (2021). Processo de enfermagem Informatizado Apoiado por Sistema Especialista na Aplicação das Escalas Brandene deGlasgow.Revista Ibérica de Sistemas e Tecnologias de Informação, 41 (02), 44-53.

Feijó, L. (2015). Avaliação do estado de consciência. Revista Universidade Porto. https://repositorio-aberto.up.pt/bitstream/10216/90400/2/37410.pdf..

GCS. Eyes Verbal Motor. (2015). A abordagem estruturada de glasgow para avaliação da escala de coma de glasgow.Sir Graham Teasdale. https://www.glasgowcomascale.org/.

Gil, A. C. (2017) Como elaborar projetos de pesquisa. Atlas (6a ed.),

Koizumi, M. S. (1978). Avaliação do nível de consciência em pacientes com traumatismo cranio-encefalico.Revista Brasil Enfermagem. 31 (1),.23-31. https://www.scielo.br/scielo.php?script=sci_arttext\&pid=S0034-71671978000100023.

Melo, J. R. T.; Silva, R. A. \& Junior, E. D. M.; (2004). Características dos pacientes com trauma cranioencefálico na cidade do Salvador, Bahia, Brasil. Revista Arquivos Neuropsiquiatria. 62 (3-A), 711-715. https://www.scielo.br/pdf/anp/v62n3a/a27v623a.pdf.

Melo, T. C; et al. (2019). Utilização da escala de coma de Glasgow para detecção precoce de complicações. In: IX Congresso Gaúcho de Terapia Intensiva, FAURGS, Gramado, RS.

Miguel, P. A. (2012). Metodologia de pesquisa para engenharia de produção e gestão de operações. Rio de Janeiro: Elsevier, ABEPRO, $2^{\text {a }}$ Ed.

Morgado, F. L. \& Rossi, L. A.; (2011). Correlação entre a escala de coma de glasgow e os achados de imagem de tomografia computadorizada em pacientes vítimas de traumatismo cranioencefálico. Radiologia Brasil.44(1):35-41. https://www.scielo.br/pdf/rb/v44n1/10.pdf.

Munizi, E. C. S.; Thomazi, M. C. A.; Kubotai; M. Y.; Cciancii, L.\& Sousa, R. M. C. Utilização da escala de coma de glasgow e escala de coma de jouvet para avaliação do nível de consciência. http://www.ee.usp.br/reeusp/upload/html/417/body/v31n2a10.htm.

Prado, C.; (2001). Ensino-aprendizagem da escala de coma de glasgow - Analise de duas técnicas em enfermeiros dos serviços de emergências. Digital Library - USP. https://www.teses.usp.br/teses/disponiveis/7/7135/tde-30032007-115512/publico/Claudia_Prado_ME.pdf.

Rezer, F; Pereira, B. F. O; Faustino. W. R. (2020). Conhecimento de enfermeiros na abordagem à vítima de traumatismo cranioencefálico. Journal Health NPEPS, 5 (2), 291-302.

Rodrigues, C. A. F \& Varanda, E. M. G. (2019). Aplicação de um programa de estimulação multissensorial a doentes com alterações severas do estado de consciência. RPER, 2 (2), $5-11$.

Ruy, E. L.\& Rosa, M. I.; (2011). Perfil epidemiológico de pacientes com traumatismo crânio encefálico. Revista Arquivos Catarinenses de Medicina. 40, (3,). <http://www.acm.org.br/acm/revista/pdf/artigos/873.pdf>.

Santos, W. C; et al. (2016). Avaliação do conhecimento de enfermeiros sobre a escala de coma de Glasgow em um hospital Universitário. Einstein, 14, (2), 213-218.

Saragiotto, D. (2020). Mortes no trânsito. In: Estadão, Observatório Nacional de Segurança Viária (Onsv), em:<https://mobilidade.estadao.com.br/mobilidadecom-seguranca/mortes-no-transito-brasileiro-mata-1-pessoa-a-cada-15-minutos/>. 
Research, Society and Development, v. 10, n. 14, e48101421643, 2021

(CC BY 4.0) | ISSN 2525-3409 | DOI: http://dx.doi.org/10.33448/rsd-v10i14.21643

Settervall, C. H. C., Sousa, R. M. C. \& Silva, S. C. F. (2011). Escala de coma de glasgow nas primeiras 72 horas após trauma cranioencefálico e mortalidade hospitalar. Revista Latino-Americano Enfermagem. 19, .6-7. 〈https://www.scielo.br/pdf/rlae/v19n6/pt_09.pdf>.

Silva, L. C. A. \& Cunha, J. (2021). Importância da atualização da escala de coma de glasgow e inclusão da avaliação pupilar em sua aplicabilidade ao protocolo de manchester. Anais do III Congresso Regional de Emergências Médicas (CREMED-CO), $<$ https://periodicos.univag.com.br/index.php/cremed/article/view/1481/1630>.

Sousa, R.; Regis, F. \& Koizumi, M. (1998). Traumatismo crânio-encefálico: diferenças das vítimas pedestres e ocupantes de veículos a motor. Depto de Enfermagem Médico-Cirúrgica da Escola de Enfermagem da Universidade de São Paulo. São Paulo, SP. <https://www.scielosp.org/article/rsp/1999.v33n1/8594/pt/>

Souza, J. C. M; et al. (2020) As entrelinhas da literatura no tocante ao uso da escala de coma de Glasgow por enfermeiros (2016). In: Geração de conhecimento nas ciências médicas: impactos científicos e sociais. Editora Amplla, 450 p. 